\title{
Valorización y defensa de la biodiversidad como fuente de desarrollo humano
}

\author{
Katia Saro', Pablo Coba Santamaría2,*
}

I Dip. Biología ed Evoluzione. Sezione di risorse agrotecnologiche e Farmaceutiche, Università degli Studi di Ferrara. Ferrara, Italia.

2 Centro de Investigación y Valoración de la Biodiversidad (CIVABI), Universidad Politécnica Salesiana, Quito, Ecuador.

* Autor para correspondencia: pcoba@ups.edu.ec

\section{Resumen}

La Universidad de Ferrara UNIFE, el Voluntariado para el Desarrollo VIS y la Universidad Politécnica Salesiana UPS del Ecuador, trabajan conjuntamente en la valoración y defensa de la biodiversidad en la Amazonía ecuatoriana, específicamente en la provincia de Morona Santiago. En este proceso la UPS salvaguarda el conocimiento ancestral de comunidades Shuar y Achuar a través de el programa académico "Tecnología en Procesamiento de Recursos Biológicos Amazónicos", complementando de esta manera la investigación y transferencia técnico-científica con el conocimiento milenario de estas etnias. Asimismo, este trabajo se ha realizado con la intervención del Centro de Investigación y Valoración de la Biodiversidad CIVABI a través de los análisis químicos de las muestras vegetales encontradas. Nuestros principales beneficiarios son los estudiantes de las comunidades shuar y achuar, quienes además de formarse en las áreas científicas, técnicas y humanas brindan soluciones eficaces a las demandas de sus comunidades.

Palabras Clave: biodiversidad, investigación, universidad, shuar, achuar, cooperación.

\section{Introducción}

La biodiversidad se ha convertido en un tema necesario de discusión actual que gana connotación debido al gran impacto que en los últimos años el ser humano ha producido en la naturaleza. Solbrig define la diversidad biológica o biodiversidad como la propiedad de las distintas entidades vivas de ser variadas (Soldbrig, 1991); es decir, que al paso de millones de años estas especies han ido adaptándose dependiendo de las condiciones medioambientales que se les han ido presentando con el transcurso del tiempo, $y$ producto de las constantes adaptaciones o mutaciones que produjeron un sinnúmero de especies entre raras y dominantes.

Así pues, la biodiversidad se evidencia en la gran variedad de seres vivos que en cada reducto de bosque, selva o cualquier ecosistema, se asientan. La conversión de bosques, selvas, páramos que se des- tinan para actividades agropecuarias o aéreas urbanas dejan resultados inevitables en el exterminio de muchas especies de plantas, animales e incluso de microorganismos.

La aplicación de la taxonomía como una herramienta para clasificar e identificar tanto especies de animales, vegetales y microorganismos es sin duda, una alternativa para conocer el impacto que la vida humana tiene con el planeta. Se ha descrito y clasificado alrededor de 1.77 millones de especies (Nebel et al., 1999) pero los científicos opinan que hasta 30 millones aún no se han clasificado. No cabe duda que es responsabilidad de todos el conservar y proteger este patrimonio, debido a esto la Universidad de Estudios de Ferrara (Italia) y la Universidad Politécnica Salesiana del Ecuador trabajan conjuntamente en la elaboración de una base de datos con la finalidad de catalogar las plantas medicinales de la región amazónica ecuatoriana. 


\section{Saro y Coba Santamaría}

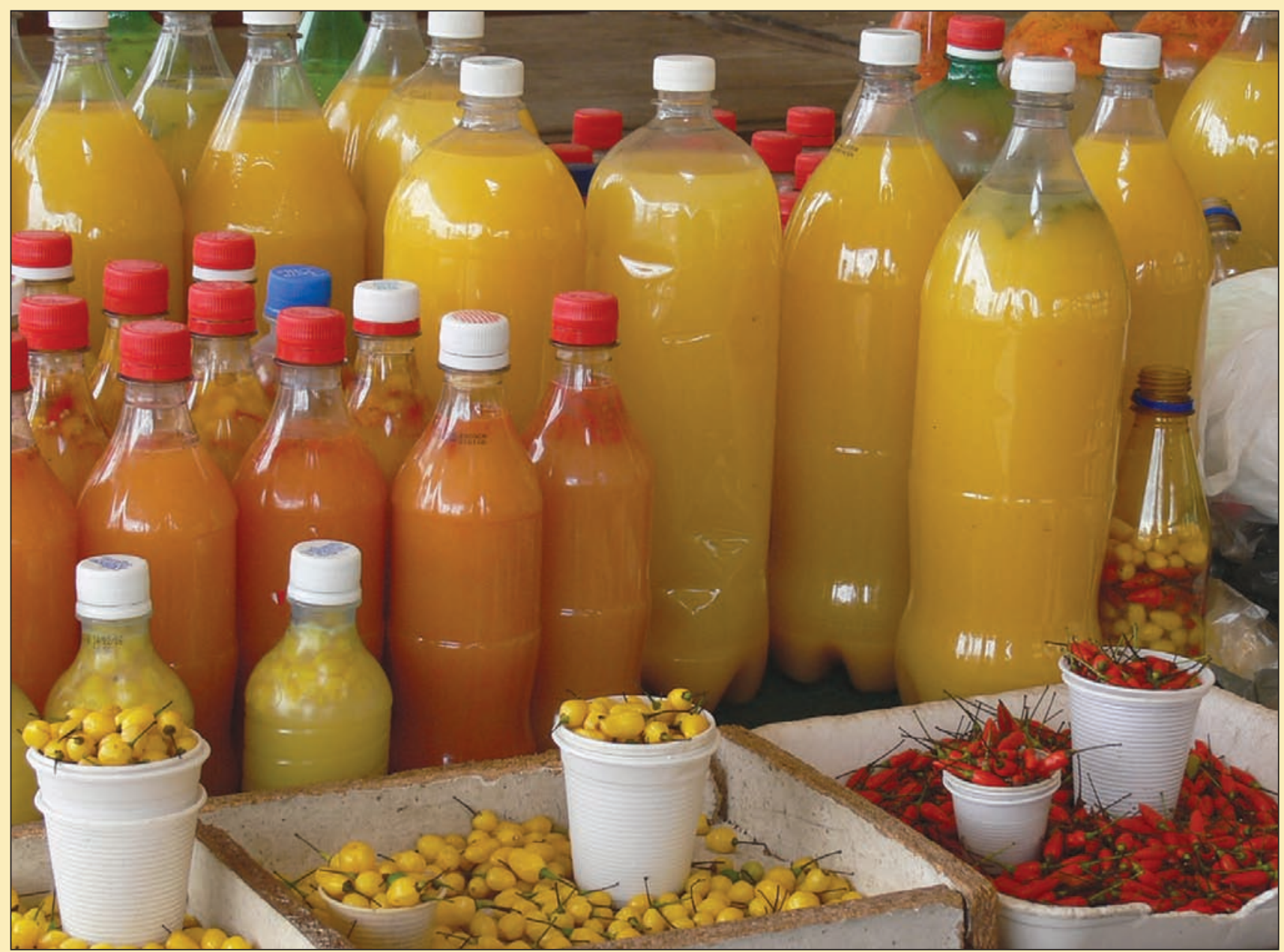

Desgraciadamente mucho del conocimiento etnobotánico de los indígenas ha sido objeto de biopiratería, que ha beneficiado a grandes intereses económicos particulares, sea con el uso directo de las especies vegetales, o con la extracción de sus recursos biogenéticos. Una manera para solucionar este problema, sería generar un registro de datos al alcance de las comunidades indígenas que garantice que los beneficios de este conocimiento ancestral llegue a manos de las propias comunidades donde se generó el conocimiento.

\section{Construcción del banco de datos}

La Universidad de Ferrara forma parte del proyecto de cooperación entre el Voluntariado Internacional para el Desarrollo VIS y la Universidad Politécnica Salesiana del Ecuador promoviendo la investigación de los recursos biológicos amazónicos desde el año 1996.

En 1999 se empezó a trabajar en el proyecto de valoración de la biodiversidad financiado con fondos del Ministerio de Asuntos Exteriores de Italia (MAE) en la ciudad de Macas, el cual concluyó en el año 2002. Como resultado se obtuvo la capacitación en temas de diversidad a medio centenar de estudiantes de la zona. En el 2005 , se planteó la necesidad de dar continuidad a este trabajo y se financió un segundo proyecto que culminará en julio de 2009.

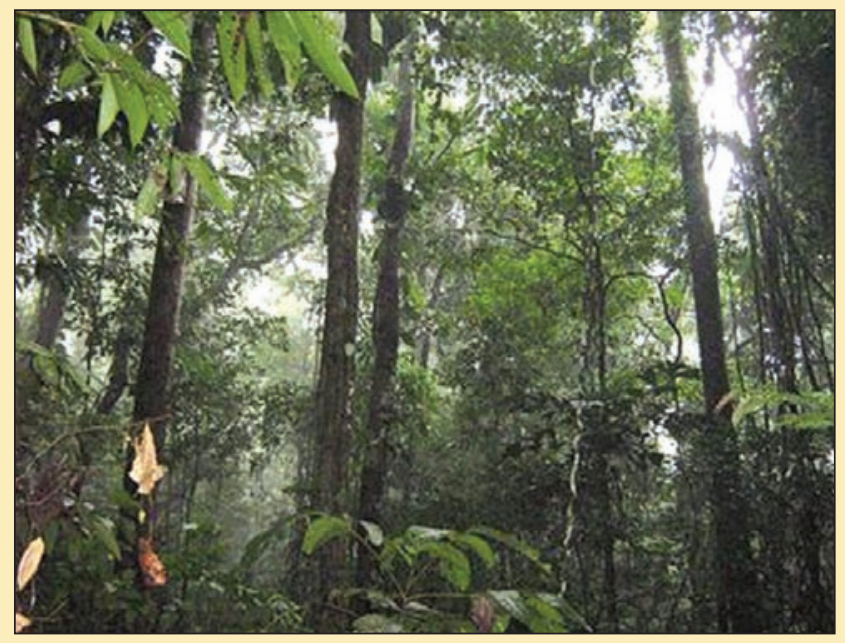




\section{La Granja 27}

Como fruto de estos proyectos, las instituciones cooperantes apoyaron a la construcción de una entidad que promueva, divulgue e investigue en temas referentes a la biodiversidad, y así, se crea el Centro de Investigación y Valorización de la Biodiversidad, CIVABI en la UPS en el año 2004.

Un último proyecto tiene como objetivo la capacitación de los indígenas amazónicos en la investigación de especies botánicas con alto interés de uso alimenticio, cosmético y farmacéutico, lo que generará que los beneficios económicos de este conocimiento sean empleados por las comunidades. Dicha investigación prevé la implementación de un herbario de especies nativas y el posterior estudio químico y biológico de las mismas, este estudio será realizado por el CIVABI contribuyendo a la valoración de la diversidad del Ecuador respetando la propiedad intelectual.

\section{Funciones del CIVABI}

En el trascurso de estos años, el CIVABI ha dado servicio a instituciones públicas y privadas, así como a clientes individuales que requieran análisis de suelos, aguas, alimentos y productos naturales así como asistencia técnica y capacitación.
La universidad en su proceso de apoyo a la investigación evalúa la actividad biológica que tiene la flora de Morona Santiago, como resultado se obtuvo el levantamiento etnobotánico de alrededor de 1000 especies de plantas de las cuales 35 especies se encuentran en un proceso avanzado de estudio. Dicha investigación se llevó a cabo con los estudiantes de Biotecnología y con el apoyo de las comunidades Shuar y Achuar, además de contar con el soporte académico de los profesores de la UPS y de los científicos del Botanical Garden de Missouri.

Las instituciones involucradas han colaborado activamente en el proceso de puesta y ejecución del proyecto invirtiendo en tecnología, y poniendo a disposición lo mejor de su material humano que se manifiesta a través de voluntarios, investigadores, cooperantes y docentes.

Todo esto sea para defender el pulmón verde más grande del planeta, la selva amazónica, y promover el desarrollo sustentable de sus comunidades gracias a la valoración de su biodiversidad.

\section{Referencias}

Nebel J Bernard y Wright T. Richard 1999. Ciencias Ambientales: Ecología y desarrollo sostenible. Edición en Español pp. I0-13 


\section{Saro y Coba Santamaría}

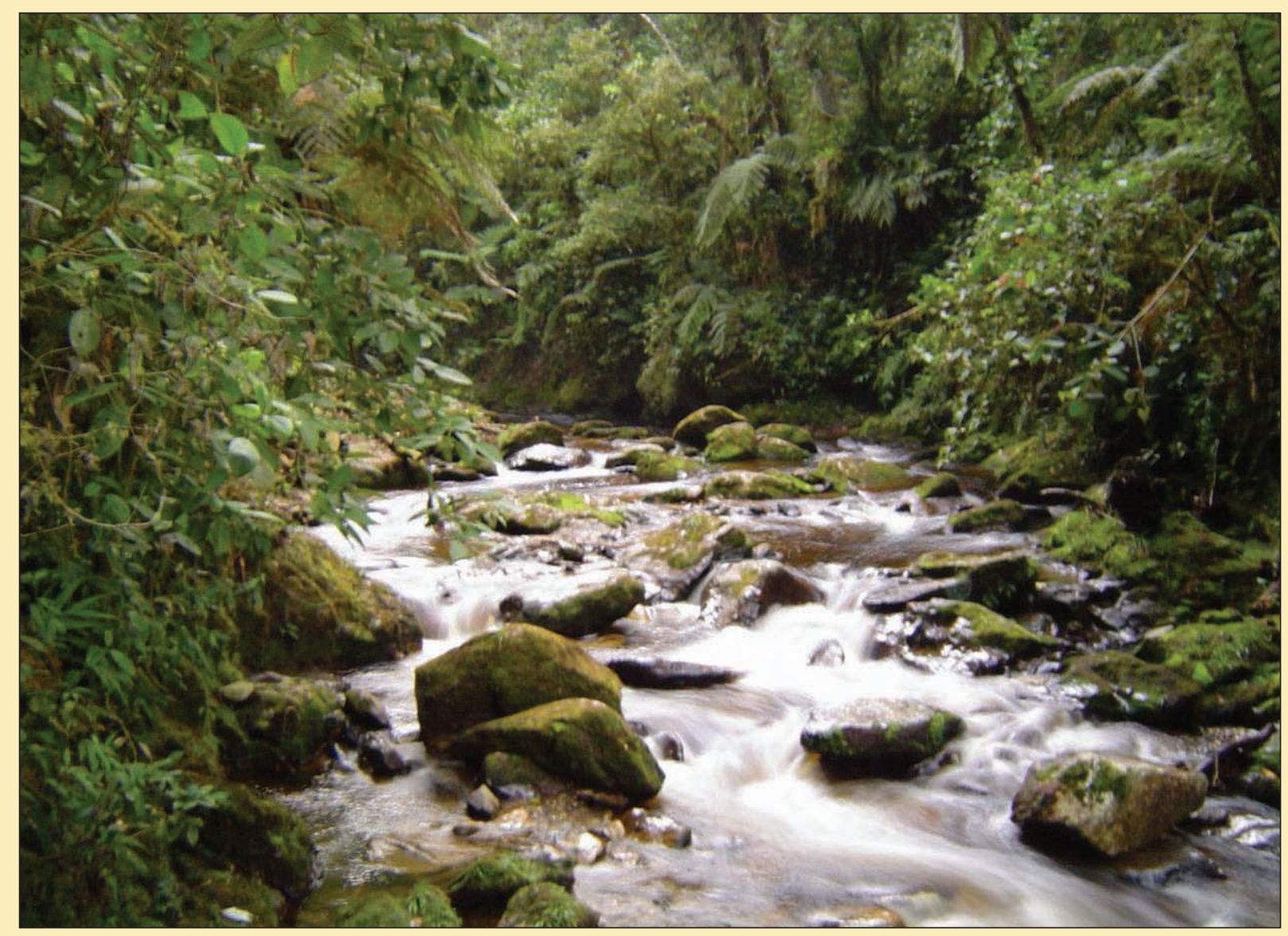

Todo esto sea para defender el pulmón verde más grande del planeta, la selva amazónica, y promover el desarrollo sustentable de sus comunidades gracias a la valoración de su biodiversidad. 